\title{
BINARY IMAGE WATERMARKING THROUGH BIASED BINARIZATION
}

\author{
Haiping Lu, Alex C. Kot \\ School of Electrical and Electronic Engineering \\ Nanyang Technological University \\ Singapore 639798
}

\author{
Rahardja Susanto \\ Institute for Infocomm Research \\ 21 Heng Mui Keng Terrace \\ Singapore 119613
}

\begin{abstract}
This paper presents a watermarking algorithm for binary images. The original binary image is blurred to a gray-level image and we embed the watermark by biasing the threshold in binarization. A loop is used to control the quality of watermarked images and robustness, and a key is generated for extraction. We employ error correction codes to reduce extraction error. This algorithm can be applied to general binary images except dithered images. Experiments show that the distortion in the watermarked image is not obtrusive and the algorithm provides some degree of robustness.
\end{abstract}

\section{INTRODUCTION}

Today, digital media are getting more and more popular. Not only multi-level images, video and audio are in digital form, but binary images are also digitized in the applications including legal documents, digital books, maps, and architectural and electronic drawings. Digital watermarking techniques have been proposed for ownership protection, copy control, annotation and authentication of digital media. Most of digital image watermarking techniques in the literature are proposed for gray-scale/color images, while the digital watermarking for binary images are only addressed by a few authors $[1,2,3,4,5]$.

Chen et al. [2] classify watermarking and data hiding techniques for binary images into various embedding methods: text line, word or character shifting, boundary modification, fixed partitioning of the image into blocks, modification of character features, modification of run-length patterns, or modification of half-tone images. Among those that are applicable to general binary images, only the algorithm proposed by Zhao and Koch [6] provides some robustness against noise while the quality of the watermarked image is poor. The algorithm alters the numbers of black and white pixels in each $8 \times 8$ block to embed data. Bit ' 1 ' is embedded if the percentage of white pixels is greater than a given threshold ( $55 \%)$ and bit ' 0 ' is embedded if the percentage of the white pixels is less than another threshold (45\%). Such modification could result in very poor marked image.
In [1], a watermark is embedded in the DC components of Discrete Cosine Transform (DCT) for binary images. It can be seen from the paper that DC component modification is a spatial domain technique in effect. Modification of DC components is equivalent to add constant values to all pixels in the spatial domain.

In this paper, we propose a more efficient watermarking algorithm for binary images other than dithered images based on the work in [1]. The original binary image is blurred to a gray-level image to enable embedding. However, DCT and IDCT (inverse DCT) are not involved, which greatly simplifies the watermarking procedure. The embedding is done by using the watermark information to bias the binarization threshold. A loop is used to control the quality of watermarked binary image and a feature vector is extracted as a key to be used in extraction so that the original binary image is no longer necessary in extraction. To improve the extraction accuracy, the watermark is coded in error correction code (ECC). Experimental results have shown that the watermarked binary image has good quality and the algorithm offers some robustness.

\section{THE PROPOSED WATERMARKING ALGORITHM}

Fig. 1 shows the system flow of the proposed algorithm. We embed the watermark $w$ into the original binary image $f(x, y)$ to obtain the output image $g(x, y)$. The image is of size $M \times N$.

\subsection{Blurring}

It is shown in [1] that it is not possible to successfully embed a watermark directly into a binary image through binarization no matter what threshold is used. Blurring is necessary to produce pixels with various intensities so that the embedding can succeed through biased binarization.

We have observed that the algorithm in [1] is likely to produce obtrusive noises near the edges. The cause is that when the $5 \times 5$ Gaussian low-pass filter processes the pixels near the edges where the $5 \times 5$ window is only partially 


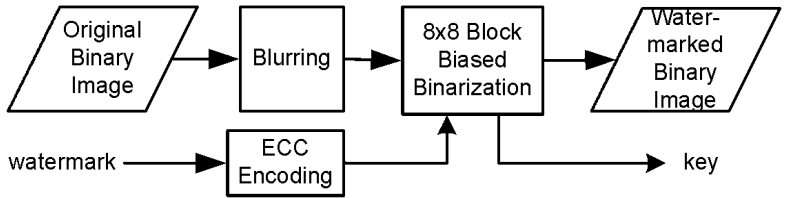

(a) Watermark embedding

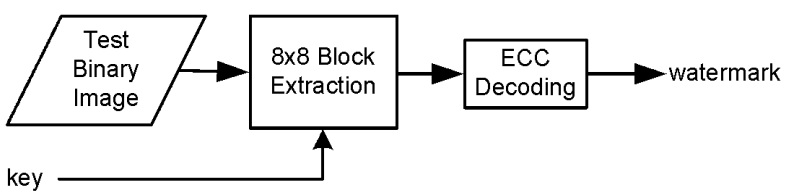

(b) Watermark extraction

Fig. 1. Binary image watermarking through biased binarization.

within the image, the part of the window that is outside of the image is treated as with pixel value 0 (black). However, black pixels near the edges are obvious against the white background. To solve this problem, we expand the original image by 2 pixels with the background color (white) for the four edges before blurring.

The original image $f(x, y)$ is expanded with white pixels (two at each edge) to an image $f_{e}(x, y)$ of size $(M+$ $2) \times(N+2) . f_{e}(x, y)$ is then low-pass filtered using a Gaussian filter with window size of $5 \times 5$ and standard deviation of 0.7 to produce $f_{e b}(x, y)$, from which the blurred version $f_{b}(x, y)$ is obtained by ignoring 2 pixels at each edge. Larger standard deviation offers better robustness but poorer quality, and vice versa. The blurred image $f_{b}(x, y)$ is then split into non-overlapped blocks of $8 \times 8$.

\subsection{Watermarking through biased binarization}

We skip the blocks in $f_{b}(x, y)$ corresponding to the uniform (all black/white) blocks in $f(x, y)$ to preserve the quality of the image after embedding. The watermark is embedded by binarizing the blocks in $f_{b}(x, y)$ that correspond to the nonuniform $8 \times 8$ blocks in $f(x, y)$ with biased thresholds. We denote the number of non-uniform $8 \times 8$ blocks in $f(x, y)$ as $N_{n}$ and each corresponding block in $f_{b}(x, y)$ as $f_{b \_k}(r, s)$, where $r, s=0,1, \ldots, 7$, and $k=0,1, \ldots, N_{n}-1$.

The watermark $w$ is a bit stream of ' 0 's and ' 1 's, instead of a random number sequence as in [1], and it is encoded with $\mathrm{BCH}(31,6)$ [7] to reduce the extraction error. The coded watermark $w_{c}$ is of length $L_{w} \leq N_{n}$.

The algorithm in [1] requires the original image in extraction, which may not be possible in practice. In our proposed algorithm, we eliminate this limitation by extracting a key to be used in extraction. This key, $k_{n}$ is extracted as the number of white pixels in each block (both uniform and non-uniform) and it is of length $L_{k e y}$, where $L_{k e y}$ is the total number of $8 \times 8$ blocks in $f(x, y)$.

For each block $f_{b_{-} k}(r, s)$, the maximum and minimum intensities are $I_{m a x_{-} k}$ and $I_{m i n \_k}$, respectively. The bias $b_{k}$ depends on $w_{c}(k)$ as follows:

$$
b_{k}= \begin{cases}0.05, & \text { if } w_{c}(k)=1 \\ -0.05, & \text { if } w_{c}(k)=0 .\end{cases}
$$

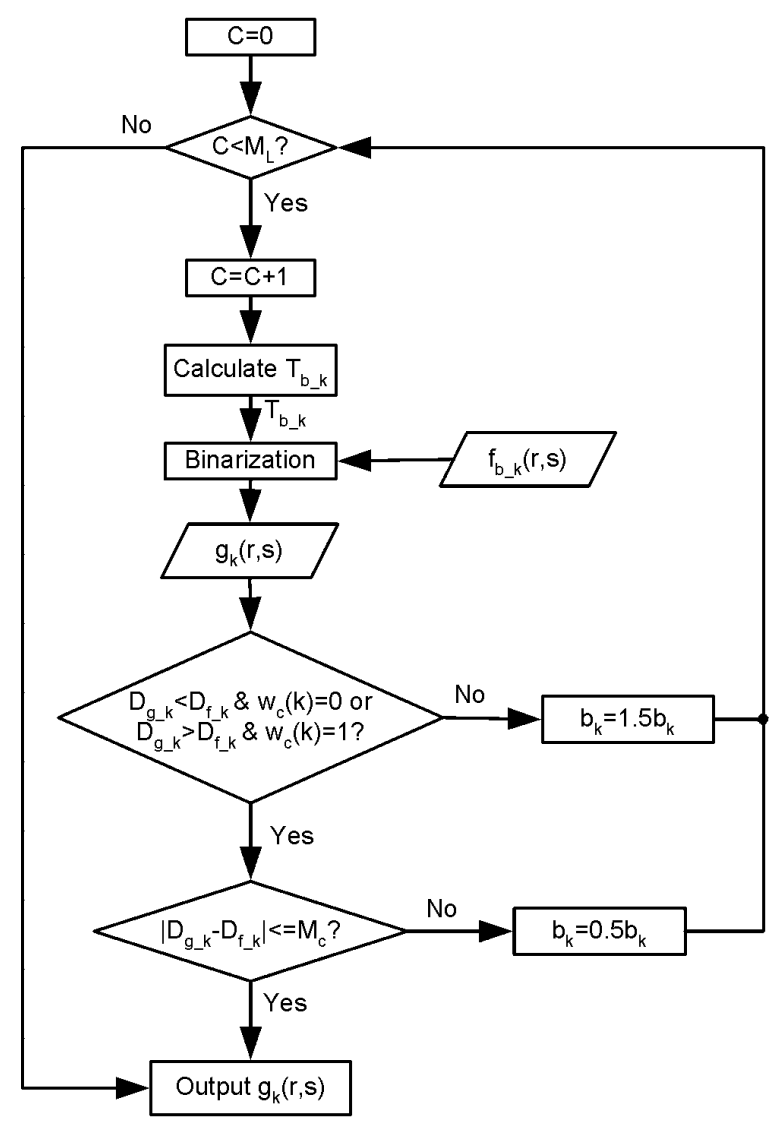

Fig. 2. Loop to control embedding.

This bias is adjusted through a loop to control the amount of distortion and the robustness, as shown in Fig. 2. $C$ is a counter and is initialized to 0 . The maximum number of iterations is limited to $M_{L}$. The binarization threshold $T_{b_{-} k}$ is calcuated as following:

$$
T_{b_{-} k}=\left(I_{\text {max } \_k}+I_{\text {min_k }}\right) \cdot\left(0.5-b_{k}\right)
$$

Thus bit ' 1 ' will lower the threshold and bit ' 0 ' will raise it. The block $f_{b_{-} k}(r, s)$ is then binarized to $g_{k}(r, s)$ using $T_{b_{-} k}$ :

$$
g_{k}(r, s)= \begin{cases}0, & \text { if } f_{b_{\_} k}(r, s)<T_{b_{\_} k} \\ 1, & \text { if } f_{b_{-} k}(r, s) \geq T_{b_{-} k} .\end{cases}
$$

Therefore, a lowered threshold (by bit ' 1 ') tends to increase the number of white (1s) pixels and a raised threshold (by bit ' 0 ') tends to reduce the number. 
We denote the number of white pixels in $f_{k}(r, s)$ and that number in $g_{k}(r, s)$ as $D_{f_{-} k}$ and $D_{g_{-} k}$, respectively. For successful embedding, we need to have the following condition:

$$
\begin{cases}D_{g_{-} k}<D_{f_{-} k}, & \text { if } w_{c}(k)=0 \\ D_{g_{-} k}>D_{f_{-} k}, & \text { if } w_{c}(k)=1 .\end{cases}
$$

If this condition is not satisfied after the biased binarization, we increase the bias to $\alpha \cdot b_{k}(\alpha>1)$ until the condition is satisfied or $C \geq M_{L}$.

On the other hand, when condition (2) is satisfied, it is possible that the distortion in the block is too much. Hence, for better quality, we should reduce the amount of bias. We denote the maximum acceptable number of changed pixels in a block as $M_{c}$. Thus we reduce the bias to $\beta \cdot b_{k}(0<$ $\beta<1)$ if $\left|D_{g_{-} k}-D_{f_{-} k}\right|>M_{c}$. Larger $M_{c}$ provides better robustness while poorer quality.

In our experiments, we choose $\alpha=1.5$ and $\beta=0.5$. Inappropriate values of $\alpha$ and $\beta$ may require more iterations.

In case that condition (2) is not satisfied after the iterations, we keep $g_{k}(r, s)=f_{k}(r, s)$, where $f_{k}(r, s)$ is the corresponding non-uniform $8 \times 8$ block in $f(x, y) . g(x, y)$ is obtained by replacing $f_{k}(r, s)$ in $f(x, y)$ with $g_{k}(r, s)$.

\subsection{Watermark extraction}

Watermark extraction is a simple process. To extract watermark $\hat{w}_{c}$ from a test binary image $g(x, y)$, we split $g(x, y)$ into $8 \times 8$ blocks. The key $k_{n}$ is required and each element in $k_{n}$ corresponds to an $8 \times 8$ block in $g(x, y)$. If the element from $k_{n}$ is either 0 or 64 , the corresponding blocks in $g(x, y)$ are skipped since there is no embedding in uniform blocks. Otherwise, a ' 1 ' is extracted if the number of white pixels in the block is greater than the value of the element from $k_{n}$, and a ' 0 ' is extracted otherwise.

\section{EXPERIMENTAL RESULTS}

We use the image in Fig. 3 as the original image $f(x, y)$. Its size is $512 \times 512$.There are $2512(\approx 61 \%)$ non-uniform $8 \times 8$ blocks out of 4096 in $f(x, y)$. Thus the key length $L_{\text {key }}=4096$ and $N_{n}=2512$. We choose $M_{L}=20$ and $M_{c}=2$ in our experiments. We generate a random bit stream $w$ of length 486 and after $\mathrm{BCH}(31,6)$ coding we have the watermark $w_{c}$ of length $L_{w}=2511$ for embedding.

The image after embedding is shown in Fig. 4. There are $3268(\approx 1.2 \%)$ pixels flipped in the image. Although the measured quality is not very good, $P S N R=19.04 d B$ and $D R D=0.58$ with $m=5$ [8], visual inspection shows that the watermarked image is still in good quality and distortion is not obtrusive. Fig. 5 shows the pixels changed due to the watermark embedding. The original binary image is brightened and the pixels flipped are shown in black dots. We can
II - L'IMPLANTATION GEOGRAPI

L'organisation de l'entreprise frar 20 régions. Des calculateurs ont importantes. On trouve ainsi des $m:$ a Lille, Bordeaux, Toulouse et $M_{1}$ Bull 300 TI à programmes cablés réglons de Nancy, Nantes, Limoges pour la comptabilité téléphonique. A l'avenir, si la plupart des fichiers etre gérés en temps différé, un cert: cessibles, voire mis a jour en ten abonnes, le fichier des renseigner abonnés contiendront des quantités । Le volume total de caractères à $\xi$ melnwe znn nnn ohonnbe a btb ae

Fig. 3. Original binary image.

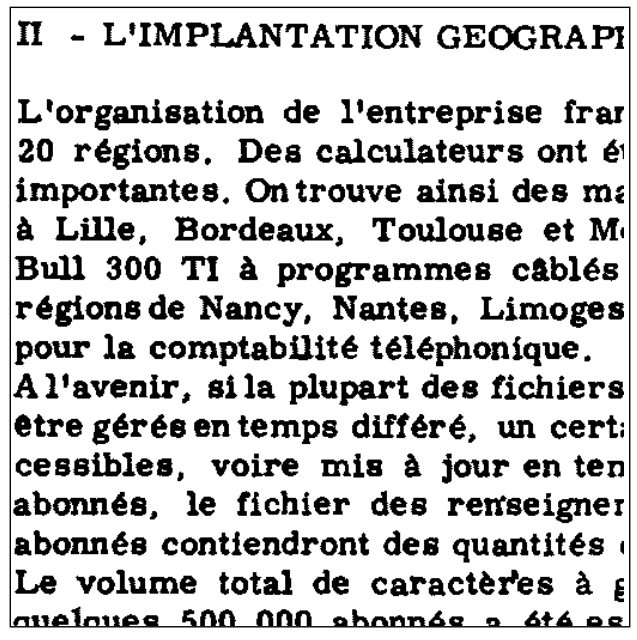

Fig. 4. Binary image after embedding.

see that most of pixels flipped are near the contours of the characters. Thus the embedding does not affect the quality of the document image much.

There is no error in the decoded $\hat{w}$ after the extraction of $\hat{w}_{c}$, attributing to the $\mathrm{BCH}$ coding. There are 147 bits error in $\hat{w}_{c}$.

The robustness against cropping and noise are shown in Fig. 6 and Fig. 7, respectively. The cropping test is implemented as in [9], where a number of rows are cropped from the watermarked image and the cropped portion is inserted into the original image to extract the watermark. The robustness against noise is tested by adding Gaussian white noise of mean 0 and variances ranging from 0.01 to 0.1 . The image after adding noise is gray and needs to be binarized. The ratio of pixels changed in the watermarked binary image before and after adding noise is shown by the dash-dot line in Fig. 7. We can see that the ECC coding, $\operatorname{BCH}(31,6)$, 
is more effective with random noise than with cropping, and its effectiveness (improvement) decreases as the amount of noise increases.

\section{CONCLUSIONS}

We propose a watermarking algorithm for binary images except dithered images. The original binary image is blurred to a gray-level image to enable embedding, and the watermark bit stream is then embedded by biasing the binarization threshold. A loop is used to control the quality of watermarked image and the robustness. A key is extracted for watermark extraction and the original binary image is not required in extraction. For higher extraction accuracy, error correction code is used. Experimental results show that the distortion in watermarked binary image is not obtrusive and the algorithm has some robustness against cropping and noise.

\section{REFERENCES}

[1] H. Lu, X. Shi, Y. Q. Shi, A. C. Kot, and L. Chen, "Watermark embedding in DC components of DCT for binary images," in Proc. IEEE Int. Workshop on Multimedia Signal Processing, US Virgin Islands, Dec. 2002.

[2] M. Chen, E. K. Wong, N. Memon, and S. Adams, "Recent developments in document image watermarking and data hiding," in Proc. SPIE Conf. 4518: Multimedia Systems and Applications IV, Aug. 2001, pp. 166-176.

[3] M. Wu, E. Tang, and B. Liu, "Data hiding in digital binary image," in Proc. of IEEE Int. Conf. on Multimedia and Expo, New York City, July 31 to August 2, 2000, pp. 393-396.

[4] Q. G. Mei, E. K. Wong, and N. D. Memon, "Data hiding in binary text documents," in Proc. of SPIE Security and Watermarking of Multimedia Contents III, Aug. 2001, vol. 4314, pp. 369-375.

[5] Y. C. Tseng, Y. Y. Chen, and H. K. Pan, "A secure data hiding scheme for binary images," IEEE Transactions on Communications, vol. 50, no. 8, pp. 1227-1231, Aug. 2002.

[6] J. Zhao and E. Koch, "Embedding robust labels into images for copyright protection," in Proc. Int. Congress on Intellectual Property Rights for Specialized Information, Knowledge and New Technologies, Vienna, Aug. 1995, pp. 242-251.

[7] B. Sklar, Digital communications : fundamentals and applications, Prentice Hall, Upper Saddle River, NJ, 2001.

[8] H. Lu, J. Wang, A. C. Kot, and Y. Q. Shi, "An objective distortion measure for binary document images based on human visual perception," in Proc. Int. Conf. Pattern Recognition, Quebec, Canada, Aug. 2002, vol. 4, pp. 239-242.

[9] Y. Liu, J. Mant, E. Wong, and S. Low, "Marking and detection of text documents using transform-domain techniques," in Proc. SPIE Conf. on Security and Watermarking of Multimedia Contents, Jan. 1999, pp. 317-328.

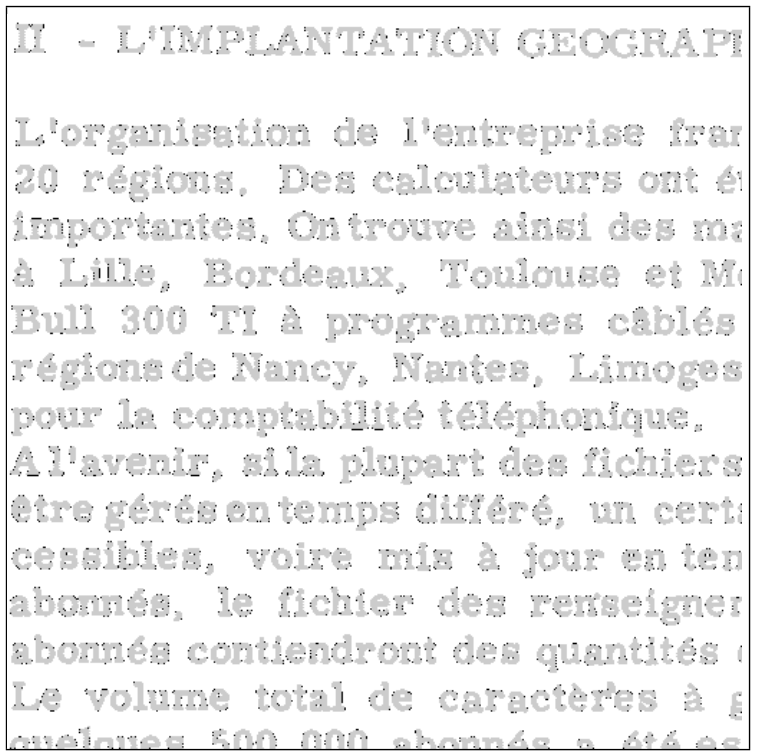

Fig. 5. Pixels flipped through watermarking.

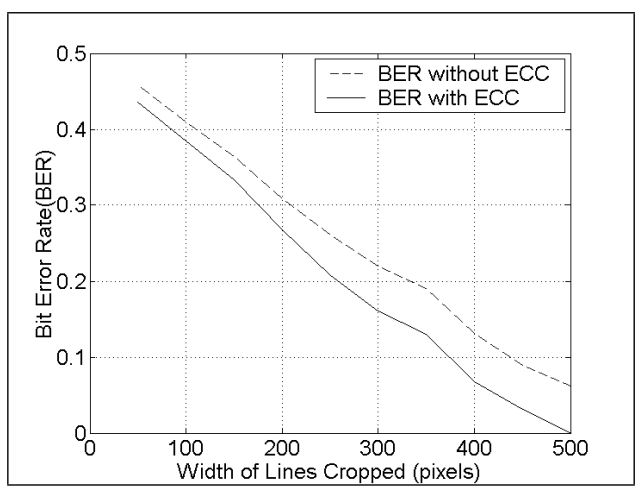

Fig. 6. Robustness against cropping.

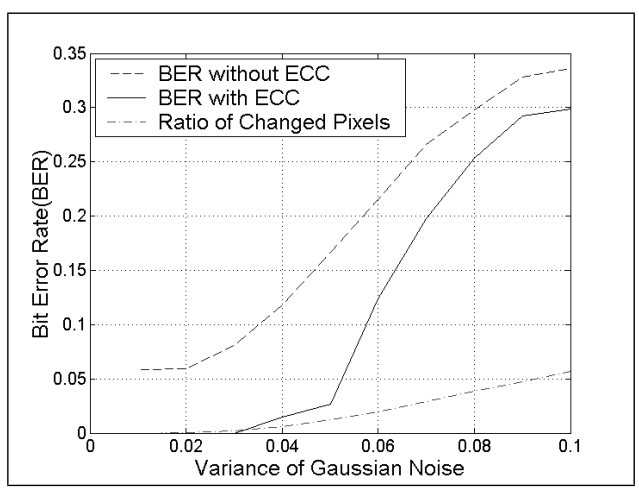

Fig. 7. Robustness against noise. 